\title{
Experimental determination of inter-sublattice exchange constants in amorphous FeGd alloys
}

\author{
Eiji Kita ${ }^{\text {a) }}$ and Yoshiaki Hata ${ }^{\text {b) }}$ \\ Institute of Applied Physics, University of Tsukuba, Tsukuba 305-8573, Japan \\ Kazuo Yano \\ C.S.T., Nihon University, Narashinodai 7-24-1, Funabashi, Chiba 274-8501, Japan \\ Hiroyuki Suzuki and Giyu Kido \\ The Tsukuba Magnet Laboratory, NIMS, Sakura 3-13, Tsukuba, Ibaraki 305-0003, Japan
}

(Presented on 7 January 2004)

\begin{abstract}
High-field-magnetization measurements were assessed for melt-spun amorphous $\mathrm{Fe}_{100-x} \mathrm{Gd}_{x}$ alloys. Samples with $X=22$ and 24 clearly revealed ferrimagnetic spin-flip transition around 100-150 kOe at $4.2 \mathrm{~K}$ and their magnetizations linearly increased with increasing magnetic field above the transition. This magnetization process with spin flipping is well understood with molecular field approximation assuming a simple two-sublattice ferrimagnet. Inter-sublattice exchange constants $J_{\mathrm{RT}}$ were obtained directly and amplitudes were $-16.5 \times 10^{-16} \mathrm{erg}$ for $X=22$ and -18.1 $\times 10^{-16}$ erg for $X=24$ samples. These agree with previously reported values estimated by molecular field analysis. () 2004 American Institute of Physics. [DOI: 10.1063/1.1682791]
\end{abstract}

\section{INTRODUCTION}

Magnetic materials composed of rare-earth (RE) and transition $(\mathrm{T})$ metal elements have been investigated from the viewpoint of both scientific interest and applications. Their amorphous alloys are the most practical of all materials because their compositions can be flexibly changed to control their physical parameters. Magnetic moments and magnetic interactions between RE and $\mathrm{T}$ elements are essential physical quantities, which influence many of their magnetic properties. It is known that $\mathrm{RE}$ elements heavier than $\mathrm{Gd}$ couple antiferromagnetically with $\mathrm{T}$ ferromagnetic elements such as Fe and Co. Therefore, the coupling between RE and $\mathrm{T}$ causes a ferrimagnetic spin structure and this is important in magnetic applications such as magneto-optical recording media. ${ }^{1}$

Magnetic interactions in amorphous RE-T alloys, $J_{\mathrm{RR}}$, $J_{\mathrm{RT}}$, and $J_{\mathrm{TT}}$, have been determined with various techniques. The first approach was where exchange constant $J_{\mathrm{RT}}$ was determined from the Curie temperature using other exchange constants. ${ }^{2}$ Here, $J_{\mathrm{TT}}$ and $J_{\mathrm{RR}}$ were estimated from $T_{\mathrm{c}}$ of "amorphous $\mathrm{Fe}$ " and the $T_{\mathrm{c}}$ of $\mathrm{RE}_{33} \mathrm{Ni}_{67}$ alloy, provided that only $J_{\mathrm{RR}}$ was held responsible for magnetic ordering. Another approach was fitting the dependence of magnetization on temperature by mean field theory. ${ }^{3}$ We investigated the exchange constants of amorphous Fe-Gd alloys (a-FeGd) by analyzing thermomagnetization curves using molecular field approximation based on a simple two sublattice ferrimagnetic model. ${ }^{4,5}$ However, in many cases, exchange constants could not be obtained directly. It is known that analyzing the high-field-magnetization process enables intersublattice interaction to be directly estimated. Using this

\footnotetext{
${ }^{a)}$ Electronic mail: kita@bk.tsukuba.ac.jp

b)Present address: Department of Applied Physics, National Defense Academy, 1-10-20 Hashirimizu, Yokosuka, Kanagawa 239-8686, Japan.
}

enabled Verhoef et al. and Liu et al. to evaluate the interaction between RE and $\mathrm{T}$ elements in ferromagnetic RE- $\mathrm{T}_{2}$ and RE-T ${ }_{3}$ intermetallic compounds. ${ }^{6,7}$ In both studies, magnetic RE elements were diluted by the nonmagnetic element of yttrium decreasing exchange coupling. There have been few reports on high-field-magnetization analysis to obtain exchange coupling in amorphous materials except for amorphous RE-Co-B alloys. ${ }^{8}$

This paper discusses high-field-magnetization measurements on melt-spun amorphous $\mathrm{Fe}_{100-\mathrm{X}} \mathrm{Gd}_{\mathrm{X}}$ alloys and the magnetic exchange constants between $\mathrm{Fe}$ and Gd we determined based on molecular field approximation.

\section{EXPERIMENT}

Amorphous Fe-Gd alloy samples (a-FeGd) were prepared with a melt-spun technique. Details on sample preparation are described elsewhere. ${ }^{9}$ The composition of samples is denoted by $\mathrm{Fe}_{100-\mathrm{X}} \mathrm{Gd}_{\mathrm{X}}$ and samples with $X$ between 22 and 60 were examined for high-field-magnetization measurements using a hybrid magnet installed at the Tsukuba Magnet Laboratories. The maximum magnetic field was $300 \mathrm{kOe}$ and a slow sweep rate enabled measurement on bulk metallic samples. ${ }^{10}$ Temperatures of the samples were fixed at $4.2 \mathrm{~K}$. A magnetic balance and a vibrating sample magnetometer (VSM) were used to obtain the dependence of magnetization on temperature for $X=22$ and 24 samples. The magnetic field applied was between 1 and $2 \mathrm{kOe}$.

The magnetization curves at $4.2 \mathrm{~K}$ are plotted in Figs. 1 and 2 for $X=22$ and 24. Magnetization curves for other samples are in Fig. 3. We can see from Figs. 1 and 2 that magnetization in samples with $X=22$ and 24 are almost constant at low magnetic fields and coercive force is quite low due to the small crystalline anisotropy from Gd. We considered that their magnetic states were in a simple ferrimagnetic state. As external field increases, magnetization clearly ex- 


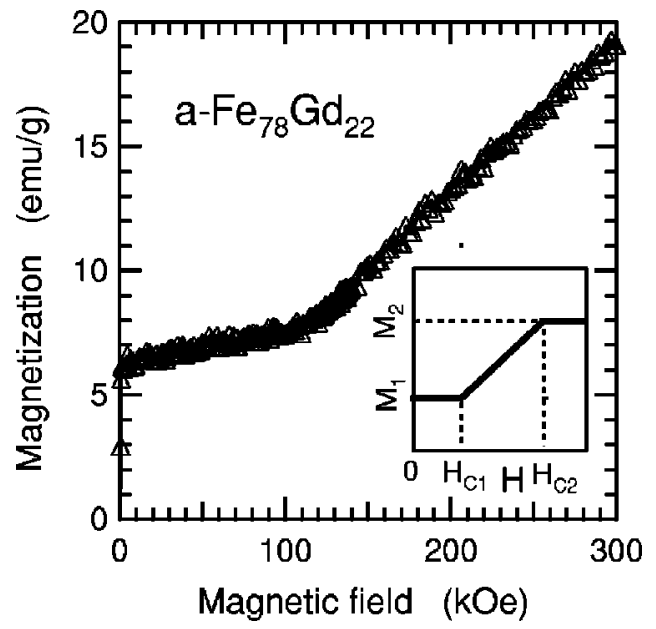

FIG. 1. High-field-magnetization curve of amorphous $\mathrm{Fe}_{78} \mathrm{Gd}_{22}$ alloy at 4.2 $\mathrm{K}$. Inset shows schematic of magnetization curve for typical ferrimagnet. Two characteristic magnetic fields have been assigned as $H_{\mathrm{c} 1}$ and $H_{\mathrm{c} 2}$.

hibited spin-flip transition and lower critical magnetic fields $H_{C 1}$ were determined to be $110 \mathrm{kOe}$ and $150 \mathrm{kOe}$. Above the critical magnetic field, magnetization increased linearly to the applied magnetic field as magnetic field increased. These magnetization curves are considered to be the spin-flip transition often seen in typical ferrimagnets. Other samples have ordinal ferromagnetic $M-H$ curves as we can see from Fig. 3. Low-field magnetization for $X \leqslant 56$ samples was well understood by the antiparallel configuration of $\mathrm{Fe}$ and $\mathrm{Gd}$ magnetic moments and the critical magnetic field for spin flipping for these samples is considered to be higher than 300 kOe, i.e., the highest magnetic field in this experiment.

The dependence of the magnetization on temperature is plotted in Fig. 4. For $X=22, T_{\mathrm{c}}$ was determined to be $540 \mathrm{~K}$. For $X=24$, compensation temperature was seen at $270 \mathrm{~K}$ and the magnetization increased rapidly at $500 \mathrm{~K}$. Crystallization started at this temperature and it is clear that $T_{\mathrm{c}}$ is higher than the crystallization temperature.

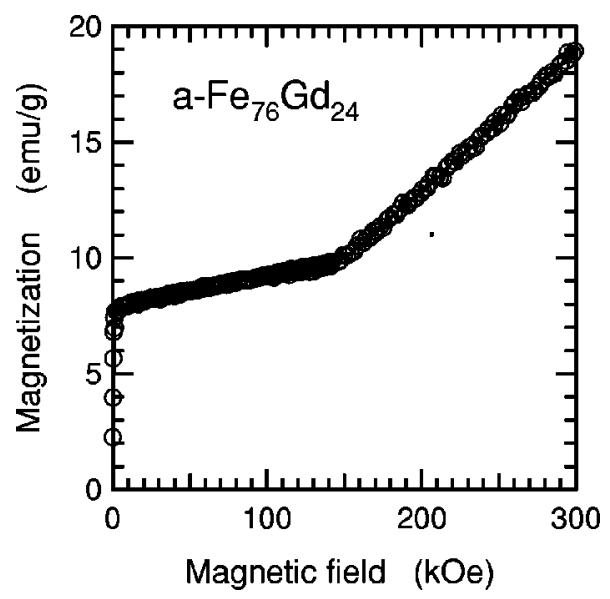

FIG. 2. High-field-magnetization curve for amorphous $\mathrm{Fe}_{76} \mathrm{Gd}_{24}$ alloy at $4.2 \mathrm{~K}$.

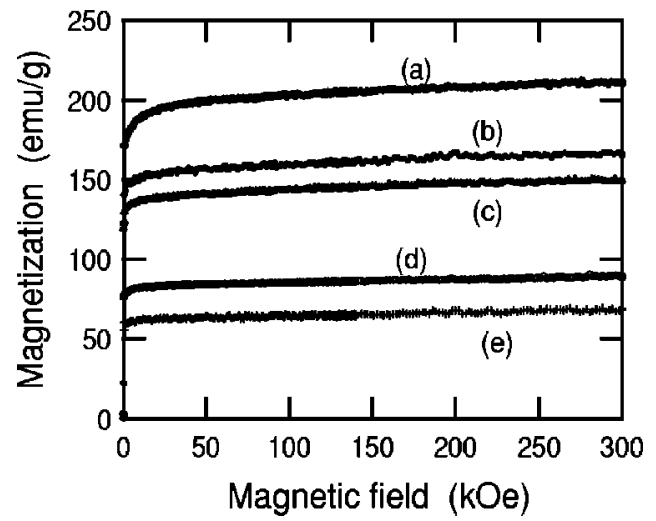

FIG. 3. High-field-magnetization curves for amorphous $\mathrm{Fe}_{100-x} \mathrm{Gd}_{x}$ alloys. Magnetization curves are for samples with $X=60$ (a), 56 (b), 52 (c), 40 (d), and $33(\mathrm{e})$.

\section{DISCUSSION}

We analyzed the spin-flip magnetization process by means of molecular field theory (MFT). The high-fieldmagnetization process for a typical two-sublattice ferrimagnet with spin-flip transition is plotted in the inset of Fig. 1. Two characteristic magnetic fields related to this transition can be defined as lower critical field $H_{C 1}$ and higher critical field $H_{\mathrm{c} 2}$. In terms of MFT, ${ }^{6,7,11}$ the exchange energy of a single RE atom $E_{\mathrm{ex}}^{\mathrm{R}}$ can be written as

$$
E_{\mathrm{ex}}^{\mathrm{R}}=-2 \sum_{\mathrm{T}} J_{\mathrm{RT}} \mathbf{S}_{\mathrm{R}} \cdot \mathbf{S}_{\mathrm{T}}=-\mathbf{m}_{\mathrm{R}} \cdot \mathbf{H}_{\mathrm{mol}}^{\mathrm{R}} \cdot
$$

Here, $J_{\mathrm{RT}}, \mathbf{m}_{\mathrm{R}}\left(=g \mu_{\mathrm{B}} \mathbf{S}_{\mathrm{R}}\right)$, and $\mathbf{H}_{\mathrm{mol}}^{\mathrm{R}}$ are an exchange constant between RE and $\mathrm{T}$ elements, a magnetic moment for a $\mathrm{RE}$ atom, and a molecular field at a RE atom site. The molecular field from $\mathrm{T}$ atoms at the RE atom site is defined as

$$
\mathbf{H}_{\mathrm{mol}}^{\mathrm{R}}=n_{\mathrm{RT}} \mathbf{M}_{\mathrm{T}}=n_{\mathrm{RT}} g \mu_{\mathrm{B}} N_{\mathrm{T}} \mathbf{S}_{\mathrm{T}},
$$

where $n_{\mathrm{RT}}$ is a molecular field coefficient and $N_{\mathrm{T}}$ is the number of $\mathrm{T}$ metal atoms. Provided that the local coordination number $Z$ is $12,{ }^{1,3}$ then $N_{\mathrm{T}}=N(100-X) / 100$ and the number

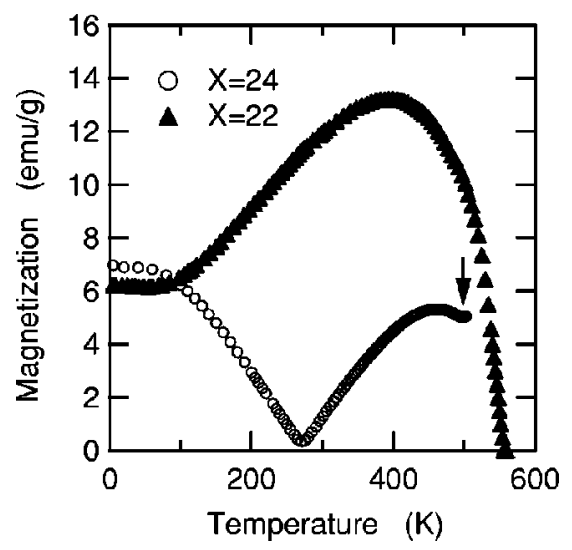

FIG. 4. Dependence of magnetization of amorphous $\mathrm{Fe}_{100-x} \mathrm{Gd}_{x}$ alloys on temperature with $X=22$ and 24 measured with VSM and magnetic balance. Arrow on $X=24$ data depicts crystallization temperature. 
TABLE I. Exchange constant $J_{\mathrm{GdFe}}$ in FeGd alloys.

\begin{tabular}{llccc}
\hline \hline & & \multicolumn{2}{c}{$J_{\mathrm{GdFe}}$} & \\
\cline { 3 - 4 } \multicolumn{1}{c}{ Sample } & & $10^{-16}(\mathrm{erg})$ & $(\mathrm{K})$ & Reference \\
\hline $\mathrm{a}-\mathrm{Fe}_{78} \mathrm{Gd}_{22}$ & Expt. & -16.5 & -12.0 & $\mathrm{a}$ \\
$\mathrm{a}-\mathrm{Fe}_{76} \mathrm{Gd}_{24}$ & Expt. & -18.1 & -13.1 & $\mathrm{a}$ \\
$\mathrm{a}-\mathrm{Fe}_{78} \mathrm{Gd}_{22}$ & $\mathrm{MFA}$ & -10.4 to & -7.5 to & 5 \\
& & -16.6 & -12.0 & \\
$\mathrm{Gd}_{0.4} \mathrm{Y}_{0.6} \mathrm{Fe}_{2}$ & Expt. & -30.4 & -22 & 7 \\
$\mathrm{Gd}_{0.75} \mathrm{Y}_{0.25} \mathrm{Fe}_{3}$ & Expt. & -19.9 & -14.4 & 7 \\
$\mathrm{GdFe}_{2}$ & Calc. & -38.8 & -28.1 & 14 \\
\hline \hline
\end{tabular}

${ }^{\mathrm{a}}$ Obtained in this work.

of $\mathrm{T}$ atoms surrounding a $\mathrm{RE}$ atom is $Z_{\mathrm{RT}}=12(100$ $-X) / 100$. The relation between $n_{\mathrm{RT}}$ and $J_{\mathrm{RT}}$ is given as follows:

$$
n_{\mathrm{RT}}=\frac{2 Z_{\mathrm{RT}} J_{\mathrm{RT}}}{g^{2} \mu_{\mathrm{B}}^{2} N_{\mathrm{T}}}=\frac{2(12) J_{\mathrm{RT}}}{g^{2} \mu_{\mathrm{B}}^{2} N},
$$

by using the number of total atoms $N\left(=N_{\mathrm{R}}+N_{\mathrm{T}}\right)$ in the sample. Then, critical magnetic fields can be expressed by $n_{\mathrm{RT}}$ and the sublattice magnetic moments of Fe and $\mathrm{Gd}$ in the sample are as follows: ${ }^{11}$

$$
H_{\mathrm{C} 1}=n_{\mathrm{RT}}\left|M_{\mathrm{Fe}}-M_{\mathrm{Gd}}\right|, H_{\mathrm{C} 2}=n_{\mathrm{RT}}\left(M_{\mathrm{Fe}}+M_{\mathrm{Gd}}\right) \text {. }
$$

$n_{\mathrm{RT}}$ can be derived from the slopes of the magnetization curves between $H_{\mathrm{C} 1}$ and $H_{\mathrm{C} 2}$, and from the $H_{\mathrm{C} 1}$ using Eq. (4). ${ }^{6}$ For example, $n_{\mathrm{RT}}$ of a- $\mathrm{Fe}_{78} \mathrm{Gd}_{22}$ was derived as 1.52 $\times 10^{4} \pm 0.02$, where $M$ was measured in emu/g units and $J_{\mathrm{RT}}$ 's were estimated as $-16.5 \times 10^{-16} \mathrm{erg}(X=22)$ and $-18.1 \times 10^{-16}$ erg $(X=24)$. Here, $m_{\mathrm{Gd}}=7 \mu_{\mathrm{B}}$ and $m_{\mathrm{Fe}}$ $=2 \mu_{\mathrm{B}}$ were used. ${ }^{5}$ The inter-sublattice exchange parameters given in the present work and estimated from research by others are summarized in Table I.

Although total local coordination number $Z$ was assumed to be 12 in this analysis, it may differ from the effective number and may affect the magnitude of the exchange constant. Petkov et al. did a detailed x-ray diffraction study on melt-spun samples prepared with the same technique as the present work. ${ }^{12}$ From Eq. (3) only total coordination number $Z$ is related when exchange constants are derived. The $\mathrm{x}$-ray experiment revealed the local coordination numbers around $\mathrm{Fe}$ and $\mathrm{Gd}$ in the $X=22$ sample were 10.4 and 11.8 , respectively. The coordination numbers for both elements were not equal and the deviation from 12 was about $15 \%$ for $\mathrm{Fe}$ and about $1 \%$ for $\mathrm{Gd}$. It is difficult to assess whether this deviation from 12 had any influence on the determination of exchange parameters. However, it can be said that errors originating from the coordination number were within $20 \%$.

We analyzed the dependence of magnetization on temperature in amorphous $\mathrm{Fe}_{100-x} \mathrm{Gd}_{x}$ alloys with MFT. ${ }^{5}$ We did least squares fitting of the thermomagnetization curve to the set of Brillouin functions by setting the three exchange parameters between $\mathrm{Fe}-\mathrm{Fe}, \mathrm{Gd}-\mathrm{Gd}$, and $\mathrm{Fe}-\mathrm{Gd}$ atoms as free parameters. However, around $X=22$, where both sublattices had almost the same magnetization, the total magnetization was very small compared with sublattice magnetization. Least squares fitting setting three exchange constants as free parameters sometimes made convergence difficult in this composition range. We therefore did fitting, where the Curie temperature was fixed and where the other two exchange parameters were free, on $X=22$ and 24 samples. The Curie temperature of the $X=24$ sample was assumed to be $550 \mathrm{~K}$ using the dependence of $T_{\mathrm{c}}$ on concentration obtained in a previous Report. ${ }^{9}$ We derived $J_{\mathrm{RT}}$ 's $-13.5 \pm 3.0$ for $X=22$ and $-13.8\left(\times 10^{-16} \mathrm{erg}\right)$ for $X=24$. These amplitudes agreed well with those obtained from the high-magnetic-field experiment.

Little theoretical evaluation of exchange coupling between RE and T elements has been carried out on amorphous states, ${ }^{13}$ while the exchange constants between Fe and Gd have been studied theoretically for compounds. Brooks et al. did an $a b$ initio calculation of molecular field interaction in RE-T intermetallics. ${ }^{14}$ Their calculations resulted in a slightly higher amplitude than that obtained in the present experiment. We can see from Table I that the exchange constants for intermetallics are larger than those for amorphous alloys determined both experimentally and theoretically. This suggests that spatial randomness presented in an amorphous state weakens exchange interaction. To extend the discussion, experimental evaluations of wider concentrations of amorphous alloys are necessary, which require a much higher magnetic field.

We determined the exchange constants between rareearth and transition metal elements without assuming other constants or $T_{\mathrm{c}}$ for melt-spun FeGd amorphous alloys. The magnitudes agreed well with those evaluated by molecular field analysis on the dependence of magnetization on temperature for the same samples.

${ }^{1}$ P. Chaudari, J. J. Cuomo, and R. J. Gambino, IBM J. Res. Dev. 17, 66 (1973).

${ }^{2}$ N. Heiman, K. Lee, and R. I. Potter, AIP Conf. Proc. 29 (1976).

${ }^{3}$ R. Hasegawa, B. E. Argyle, and L. J. Tao, AIP Conf. Proc. 24 (1975).

${ }^{4}$ K. Yano, E. Kita, K. Tokumitsu, H. Ino, and A. Tasaki, J. Magn. Magn. Mater. 104-107, 131 (1992).

${ }^{5}$ K. Yano, Y. Akiyama, K. Tokumitsu, E. Kita, and H. Ino, J. Magn. Magn. Mater. 214, 217 (2000).

${ }^{6}$ R. Verhoef, R. J. Radwanski, and J. J. M. Franse, J. Magn. Magn. Mater. 89, 176 (1990).

${ }^{7}$ J. P. Liu, F. R. de Boer, and K. H. J. Buschow, J. Magn. Magn. Mater. 98, 291 (1991).

${ }^{8}$ R. J. Radwański, J. J. M. Franse, R. Krishnan, and H. Lassri, J. Magn. Magn. Mater. 119, 221 (1993).

${ }^{9}$ K. Yano, K. Tokumitsu, E. Kita, H. Ino, and A. Tasaki, Jpn. J. Appl. Phys., Part 2 30, L482 (1991).

${ }^{10}$ H. Maeda, K. Inoue, T. Kiyoshi, T. Asano, Y. Sasaki, T. Takeuchi, K. Itoh, H. Aoki, and G. Kido, Physica B 216, 141 (1996).

${ }^{11}$ N. Miura, I. Oguro, and S. Chikazumi, J. Phys. Soc. Jpn. 45, 1534 (1978).

${ }^{12}$ V. Petkov, K. Yano, and E. Kita, Phys. Status Solidi A 157, 365 (1996).

${ }^{13}$ M. Kaburagi, O. Ozaki, and T. Tonegawa, J. Magn. Magn. Mater. 126, 307 (1993).

${ }^{14}$ M. S. S. Brooks, T. Gasche, S. Auluck, L. Nordström, L. Severin, J. Trygg, and B. Johansson, J. Appl. Phys. 70, 5972 (1991). 